\title{
A SUGGESTION FOR FINANCIAL CONDITION INDEX FOR TURKISH TOURISM SECTOR
}

\author{
DOI: 10.17261/Pressacademia.2019.1137 \\ PAP-IFC- V.10-2019(5)-p.22-26
}

Elcin Aykac Alp ${ }^{1}$, Mefule Findikci Erdogan²

${ }^{1}$ Istanbul Commerce University, Faculty of Business, Department of Economics, SutluceBeyoglu, Istanbul, Turkey. ealp@ticaret.edu.tr, ORCID: 0000-0001-9076-2102

${ }^{2}$ Istanbul Commerce University, PhD Program in Banking and Finance, Sutluca, Beyoglu Istanbul, Turkey. mfindikci@ticaret.edu.tr, ORCID: 0000-0003-0150-0990

To cite this document

Alp, E.A., Erdogan, M.F., (2019). A suggestion for financial condition index for Turkish tourism sector. PressAcademia Procedia (PAP), V.10(3), p.22-26

Permemant link to this document: http://doi.org/10.17261/Pressacademia.2019.1137

Copyright: Published by PressAcademia and limited licenced re-use rights only.

\begin{abstract}
Purpose- When the economic structures and growth behaviors of the countries are examined, it is seen that the countries with the advantage in the tourism sector have achieved significant role if they give the necessary importance to this sector. It is insufficient and incomplete to consider the issue only in terms of macroeconomic indicators. In our study, we think that this sector should take place in the plans made by considering this sector and should be followed carefully. When the world literature is examined, it is seen that tourism financial conditions index has been developed and it has taken place in finance literature. However, we believe that we need to be taking into consideration of terrorism for this index in countries like Turkey. In our study, we introduce a new index by extension with terror variable to the tourism financial conditions index which is used and developed in the literature.

Methodology- In this study, Consumer Confidence Index (CCI), Composite Leading Indicator (CLI) Consumer Price Index (CPI), Real Effective Exchange Rate (REE), number of tourist arrivals (ARV) and number of terrorist attacks (TRR) for the period between 2004:01-2017:12 are used. The Factor Analysis is used to determine the direction of the coefficients of variables. The main purpose of data normalization is to guarantee the quality of the data before it is subjected to any analysis. In the study, min-max and z score methods, which are the most commonly used statistical normalization methods, were used in the normalization of variables.

Findings- According to factor analysis findings, only terror variable affects negatively on the TFCl. Other variables affect positively on the TFCITwo composite indicators were formed from the combination of the variables obtained as a result of standardization. These two variables are different in structure. Both composite indicators are made primarily from seasonal effects of the removal process.

Conclusion- As a result, Factor analysis is used to determine the direction of the coefficients of the variables that form the index. According to factor analysis findings, only terror variable affects negatively on the TFCl. And then two separate standardization methods were used to form the composite indicator. These are Z-standardization and Min-Max method. The variables obtained with both methods have seasonal effects, also the seasonally adjusted indices are reported.
\end{abstract}

Keywords: Tourism financial conditions index, tourism sector, monetary conditions index, financial conditions index JEL Codes: E44, E47, G32

\section{INTRODUCTION}

The countries with the advantage in the tourism field have achieved important successes if they give the necessary importance to this sector. It is wrong and incomplete to consider the issue only in terms of macroeconomic indicators. In our study, we think that this sector should take place in the plans made in finance and should be followed carefully. When the literature is examined, it is seen that the tourism financial conditions index has been developed and has taken its place in the financial literature. Chang, Hsu and McAleer (2014); Chang (2015); Chang, Hsu and McAleer (2017) developed an alternative composite indicator for tourism based on $\mathrm{MCl}$ (Monetary Conditions Index) and FCl (Financial Conditions Index). The index is called the Tourism Financial Conditions Index in their studies. But we in countries like Turkey which is fighting terrorism in this study, we believe that terrorism should be calculated by adding the index of this variable. In this study, we propose a new index by adding terror variable to the tourism financial conditions index which is used and developed in the literature.

Chang (2017) proposed the Tourism Financial Conditions Index (TFCI). In this study, daily stock market returns, tourism stock market subindex returns, exchange rate returns and interest rate differences are used for Taiwan on June 1, 2001 - February 28,2014 period. TFCI was created by adapting and extending the commonly used Monetary Conditions Index ( $\mathrm{MCl}$ ) and Financial Conditions Index (FCl) to tourism 
stock data. In this study, unlike TFCl calculations in the literature, the method GARCH, GJR and EGARCH conditional volatility models are discussed to determine natural volatility in daily tourism stock market returns. As a result, the findings showed that the new tourism financial conditions index can be estimated by using the conditional average of the return of the tourism stock market index. Also, the new daily TFCl was underlined simplicity and ease of interpretation. And the index clearly expressed that it would be useful in predicting the current economic and financial environment for tourism stock market returns, especially since it is based on the calculation and interpretation of publicly available information.

\section{METHODOLOGY}

\subsection{Data}

In this study, Consumer Confidence Index (CCI), Composite Leading Indicator (CLI) Consumer Price Index (CPI), Real Effective Exchange Rate (REE), number of tourist arrivals (ARV) and number of terrorist attacks (TRR) for 2004:01- 2017:12 period data. In the study, the coefficients of variables are determined the direction of the factor analysis in the index. Then standardization methods were used and a composite index was obtained from the variables. The definitions of the variables used in the analysis are given below.

$\mathrm{CCl}$ : Consumer confidence index (This consumer confidence indicator provides an indication of future developments of households' consumption and saving, based upon answers regarding their expected financial situation, their sentiment about the general economic situation, unemployment and capability of savings)

CLI: Composite leading indicator (The composite leading indicator (CLI) is designed to provide early signals of turning points in business cycles showing the fluctuation of the economic activity around its long term potential level. CLIs show short-term economic movements in qualitative rather than quantitative terms.)

CPI: Consumer Price Index (Inflation measured by consumer price index (CPI) is defined as the change in the prices of a basket of goods and services that are typically purchased by specific groups of households)

REE: Real Effective Exchange Rate (Real effective exchange rate, it is obtained by purifying relative price effects in the nominal effective exchange rate.)

ARV: Total Visitor Arrivals

TRR: Number of terrorist attacks in the Global Terrorism Database

Standardization methods applied to variables are reported below.

\subsection{Methodology for Constructing New Index}

$\mathrm{TFCl}$, Consumer Confidence Index (CCI), Composite Leading Indicator (CLI) Consumer Price Index (CPI), Real Effective Exchange Rate (REE), number of tourists (ARV) and number of terrorist attacks (TRR) used in the study. The main purpose of data normalization is to guarantee the quality of the data before it is subjected to any analysis. There are many types of data normalization in the literature. Data normalization is particularly useful for the modelling application where data is usually of different scales. Min-max, Z-score, median normalization, etc. in the literature. Although there are many different techniques such as min-max and $z$ score methods are preferred (Jain, Nandakumar \& Ross, 2005; Jain \&Bhandare, 2011; Nayak, Misra\& Behera, 2014; Chiaramonte, Croci\& Poly, 2015; Svirydzenka, 2016; Mare, Moreira \& Rossi, 2017; Jain, Shukla \&Wadhvani, 2018). Therefore, in the study, min-max and z score methods, which are the most commonly used statistical normalization methods, were used in the normalization of variables.

The most commonly used score normalization technique is the z-score that calculated using the arithmetic mean and standard deviation of the given data. In the Z-score method, each observation data is subdivided to the standard deviation after subtraction from the observation mean to eliminate the difference in the unit of measurement of the data.

$z_{i}=\frac{x_{i}-\mu}{\sigma}$

" $\mu$ " is the arithmetic mean and $\sigma$ is the standard deviation of the given data. Z-score normalization determines a common numerical range for normalized scores of different structures.

The simplest normalization technique is Min-Max normalization. Min-Max normalization maintains the original score distribution other than the scaling factor and converts all scores into a common range. In this method, the difference between the minimum value of each observation and the series is divided by the difference between the maximum and minimum values of the series.

$s_{i}=\frac{x_{i}-x_{\min }}{x_{\max }-x_{\min }}$

In order to calculate TFCl, the different structure between the data was corrected by conversion according to variables, Min-Max and Zscore methods.

The corrections were made for all the variables used and the analysis was performed on the values that are clearly written and calculated below. 
The procedure for calculating the values obtained by the Min-Max Method is shown below,

Consumer Confidence Index : $s C C I_{i}=\frac{C C I_{i}-C C I_{\min }}{C C I_{\max }-C C I_{\min }}$,

Consumer Leading Indicator $: s C L I_{i}=\frac{C L I_{i}-C L I_{\min }}{C L I_{\max }-C L I_{\min }}$,

Consumer Price Index : $s C P I_{i}=\frac{C P I_{i}-C P I_{\min }}{C P I_{\max }-C P I_{\min }}$,

Re al Effective Exchange Rate $: s R E E_{i}=\frac{R E E_{i}-R E E_{\min }}{R E E_{\max }-R E E_{\min }}$,

Number of Tourist : $s A R V_{i}=\frac{A R V_{i}-A R V_{\min }}{A R V_{\max }-A R V_{\min }}$,

Number of Terror Attack $: s T R R_{i}=\frac{T R R_{i}-T R R_{\min }}{T R R_{\max }-T R R_{\min }}$,

The procedure for calculating the values obtained by the Z-Score Method is shown below,

Consumer Confidence Index : $z C C I_{i}=\frac{C C I_{i}-\mu_{C C I}}{\sigma_{C C I}}$,

Consumer Leading Indicator : $z C L I_{i}=\frac{C L I_{i}-\mu_{C L I}}{\sigma_{C L I}}$,

Consumer Price Index : $z C P I_{i}=\frac{C P I_{i}-\mu_{C P I}}{\sigma_{C P I}}$,

Re al Effective Exchange Rate $: z R E E_{i}=\frac{R E E_{i}-\mu_{R E E}}{\sigma_{R E E}}$,

Number of Tourist : $z A R V_{i}=\frac{A R V_{i}-\mu_{A R V}}{\sigma_{A R V}}$,

Number of Terror Attack $: z T R R_{i}=\frac{T R R_{i}-\mu_{T R R}}{\sigma_{T R R}}$,

Factor analysis is a method for investigating whether several variables of interest are linearly related to a smaller number of unobservable factors. Usually, the goal of factor analysis is to aid data interpretation. The factor analyst hopes to identify each factor as representing a specific theoretical factor. Therefore, many of the reports from factor analysis are designed to aid in the interpretation of the factors (NCSS Statistical Software, 2014). Another goal of factor analysis is to reduce the number of variables. However, Factor analysis is used in the literature to determine the weights and direction of the variables that form the index while creating composite indexes.

\section{FINDINGS AND DISCUSSIONS}

Factor analysis is performed to determine the coefficients of the series to be included in the TFCl. According to the Factor analysis findings, when creating the TFCl variable, it was determined that the coefficient of the terror variable should be negative and the coefficient of other variables should be positive.

Table 1: Factor Analysis

\begin{tabular}{|c|c|c|c|}
\hline \multicolumn{4}{|c|}{ Rotated Component Matrix } \\
\hline & 1 & 2 & 3 \\
\hline TRR & & -0.695 & \\
\hline $\mathrm{CPI}$ & & 0.893 & \\
\hline $\mathrm{CCl}$ & 0.779 & & \\
\hline $\mathrm{CLI}$ & 0.856 & & \\
\hline REE & & & 0.763 \\
\hline
\end{tabular}




\begin{tabular}{|c|c|c|}
\hline ARV & 0.694 \\
\hline \multicolumn{2}{|c|}{ Extraction Method: Principal Component Analysis. } \\
Rotation Method: Varimax with Kaiser Normalization. \\
\hline \multicolumn{2}{|c|}{ Kaiser's measure of sampling adequacy (MSA) $=0.528$} \\
\hline
\end{tabular}

After the variables were standardized, TFCl index was calculated and graphed according to both standardization techniques.

$Z$ Score $:$ TFCI $_{i}=z C C I_{i}+z C L I_{i}+z C P I_{i}+z R E E_{i}+z C C I_{i}+z A R V_{i}-z T R R_{i}$

Min-Max Score $:$ TFCI $_{i}=s C C I_{i}+s C L I_{i}+s C P I_{i}+s R E E_{i}+s C C I_{i}+s A R V_{i}-s T R R_{i}$

\section{Figure 1: TFCI Indices Obtained by Z-Score and Min-Max Standardization Technique}

TFCIZ

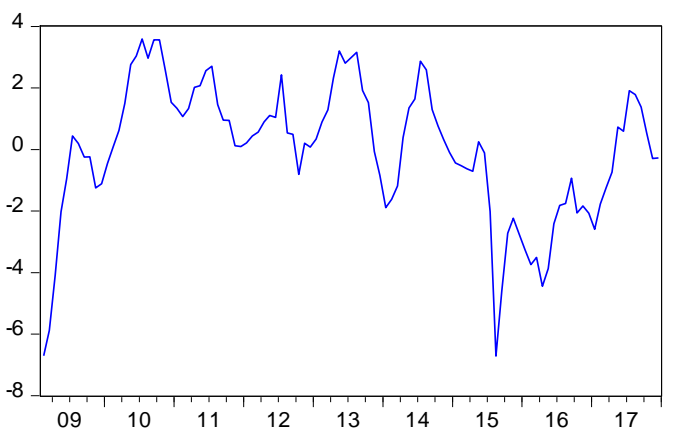

Figure 2. Seasonally adjusted $\mathrm{TFCl}$ indices

TFCIZ_SA

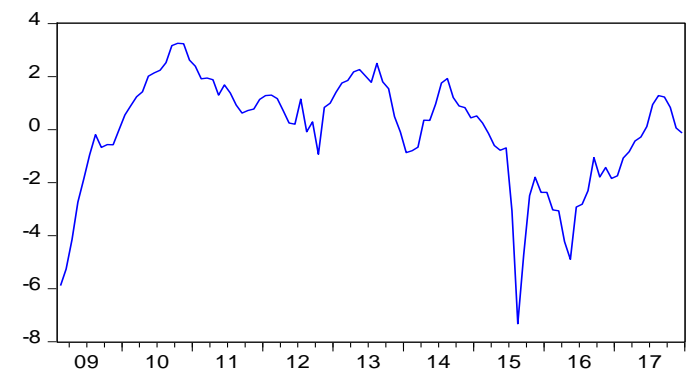

TFCIM

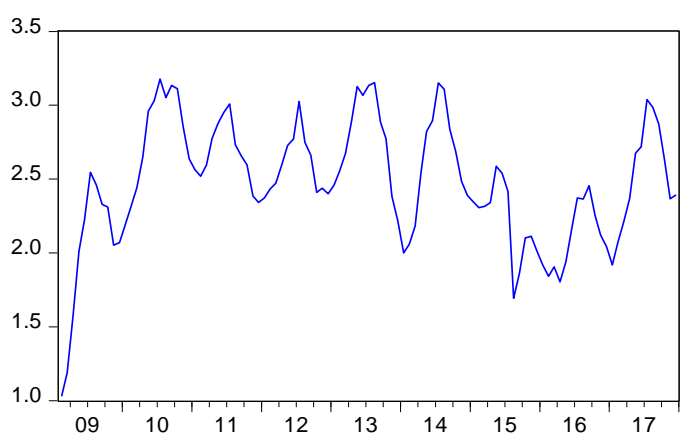

TFCIM SA

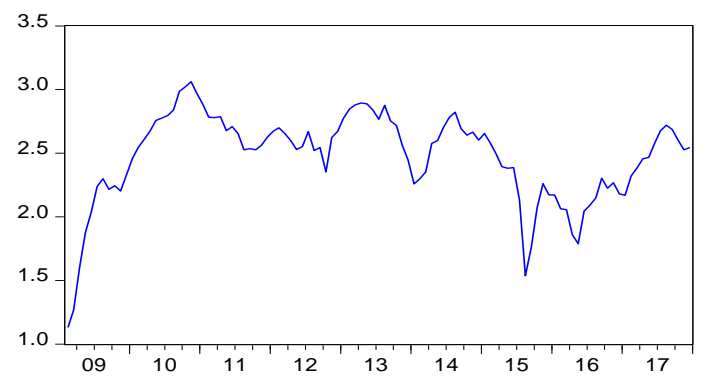

Two alternative variables were formed from the combination of the variables obtained as a result of standardization. These two variables are different in structure. Both composite indicators are made primarily from seasonal effects of the removal process.

\section{CONCLUSION}

In this study, the TFCl index introduced by Chan et.al. (2014), Chan (2015) and Chan et al. (2017) is improved by adding the effect of terrorist attacks. TFCl which has already been introduced by Chans' studies is conducted in Turkey for the period of 2004-2017. The methodology we used for this composite indicator is also revised whit transformation process. One reason for the selection of Turkey in the study of the tourism industry is affected by terrorist attacks.

First, factor analysis is used to determine the direction of the coefficients of the variables that form the TFCl. Accordingly, the analysis findings, only terror variable affects negatively on the TFCl. Then two separate standardization methods were used to form the composite indicator. These are Z-standardization and Min-Max method. The variables obtained as a result of both methods were determined for the presence of seasonal effects and seasonally adjusted.

\section{REFERENCES}

Chang, C.-L. (2015). Modelling a latent daily Tourism Financial Conditions Index. International Review of Economics \& Finance, 40, $113-126$. doi:10.1016/j.iref.2015.02.006.

Chiaramonte, L., Croci, E., \&Poli, F. (2015). Should we trust the Z-score? Evidence from the European Banking Industry. Global Finance Journal, 28, 111-131. 
Jain, A., Nandakumar, K., \& Ross, A. (2005). Score normalization in multimodal biometric systems. Pattern recognition, $38(12), 2270-2285$.

Jain, Y. K., \&Bhandare, S. K. (2011). Min max normalization based data perturbation method for privacy protection. International Journal of Computer \& Communication Technology, 2(8), 45-50.

Jain, S., Shukla, S., \&Wadhvani, R. (2018). Dynamic selection of normalization techniques using data complexity measures. Expert Systems with Applications, 106, 252-262.

Mare, D. S., Moreira, F., \& Rossi, R. (2017). Nonstationary Z-score measures. European Journal of Operational Research, 260(1), 348-358.

Svirydzenka, K. (2016). Introducing a new broad-based index of financial development. International Monetary Fund.

Nayak, S. C., Misra, B. B., \& Behera, H. S. (2014). Impact of data normalization on stock index forecasting. Int. J. Comp. Inf. Syst. Ind. Manag. Appl, 6, 357-369.

NCSS Statistical Software, Chapter 448, Fuzzy Clustering(2014, August 25). RetrievedFrom the NCSS Documentation website:

http://ncss.wpengine.netdna-cdn.com/wp-content/themes/ncss/pdf/Procedures/NCSS/Fuzzy_Clustering.pdf 